\title{
ON SOME BASIC CONSTRUCTIONS IN CATEGORIES OF QUANTALE-VALUED SUP-LATTICES
}

\author{
RADEK ŠLESINGER
}

\begin{abstract}
If the standard concepts of partial-order relation and subset are fuzzified, taking valuation in a unital commutative quantale $Q$, corresponding concepts of joins and join-preserving mappings can be introduced. We present constructions of limits, colimits and Hom-objects in categories $Q$-Sup of $Q$-valued fuzzy joinsemilattices, showing the analogy to the ordinary category Sup of join-semilattices.
\end{abstract}

\section{INTRODUCTION}

In the standard concept of a fuzzy set [11], the relation " $x$ is an element of $X$ " is fuzzified, and replaced by a mapping $X \rightarrow[0,1]$ assigning to each element of $X$ its "membership degree". As further generalization, the membership degree can be evaluated in structures more general than the real unit interval, typically frames, residuated lattices, or quantales.

In our paper, the concept of a set remains unchanged, and it will be the partial order relation and the notion of a subset that will be replaced by suitable mappings to a quantale. Instead of considering on $X$ a partial order relation $\leq$, we employ a unital quantale $Q$ and mappings $M: X \rightarrow Q$ and $e: X \times X \rightarrow Q$, which quantify the "degree of truth" of membership in a subset and of being less or equal.

Sets equipped with quantale-valued binary mappings were initially investigated in the so-called quantitative domain theory [4]. A number of papers on the topic of sets with fuzzy order relations valuated in a complete lattice with additional structure have been published in recent years. Among many others, articles $[9$, $10,12]$ may be used as a reference. There are various structures used for fuzzy valuation in the literature, e.g., frames [3] and complete residuated lattices [12], both being just special cases of quantales. Also terminology has not settled yet, and differs among authors. As the multiplicative unit of a quantale need not be its top element, even truth can have more degrees in $Q$. This is different from valuation using frames or residuated lattices where the unit is the top element as well.

The basic properties of the category of complete join-semilattices as well as the fundamental constructions in this category such as limits and colimits have

$M S C$ (2010): primary 08A72, 06F99; secondary $18 \mathrm{~B} 35$.

Keywords: sup-lattice, quantale, $Q$-order, limit, colimit.

The research was supported by the bilateral project "New Perspectives on Residuated Posets" financed by the Austrian Science Fund: project I 1923-N25 and the Czech Science Foundation: project $15-34697 \mathrm{~L}$. 
been known for a long time. Despite quite an intensive investigation in recent decades, to the author's knowledge, these constructions in categories of $Q$-valued join-complete semilattices ( $Q$-sup-lattices) have not been investigated. This paper, therefore, aims to fill this small gap.

In our paper the matter will be presented in relation to the standard concepts of the theory of posets and sup-lattices. This should convince the reader that fuzzy structures behave in strong analogy to their crisp counterparts. Throughout the text, we will try to highlight an important fact: that a fuzzy concept (e.g., subset or partial order relation) need not be treated as something more general than its crisp variant, but rather as the crisp concept satisfying an additional property.

Based on a fuzzy order relation and subset membership, fuzzy counterparts to order-theoretic concepts can be defined, such as monotone mappings, adjunctions, joins and meets, or complete lattices. After introducing these required concepts, by presenting constructions of limits and colimits, we prove that, for any commutative quantale $Q$, the category of $Q$-sup-lattices is both complete and cocomplete, and we show that it can be endowed with an internal Hom functor, which provides a new example of so-called $Q$-quantales.

\section{Preliminaries}

We just need to recall a few basic concepts related to posets, sup-lattices and quantales. The book [8] and the chapter [6] can be used as a better reference. A concise overview of constructions in the category of sup-lattices can also be found in $[5$, Chapter I].

\subsection{Posets and sup-lattices}

Two monotone mappings $f: X \rightarrow Y$ and $g: X \rightarrow Y$ are adjoint if $f(x) \leq y \Longleftrightarrow$ $x \leq g(y)$ for all $x \in X, y \in Y$ ( $f$ is a left adjoint, $g$ is a right adjoint). A right adjoint to $f$, if it exists, is unique and denoted $f^{*}$ (likewise, the left adjoint to $g$ is $g_{*}$ ).

By a sup-lattice we mean a complete join-semilattice (actually a complete lattice), with its least and greatest elements denoted by $\perp$ and $T$, respectively. Joinpreserving mappings are taken for sup-lattice homomorphisms (which makes a difference from the category of complete lattices, in which objects are the same, but morphisms are supposed to preserve both joins and meets). Preservation of arbitrary joins implies that $f(\perp)=\perp$, the join of the empty set, for any sup-lattice homomorphism $f$.

For a join-preserving map $f: X \rightarrow Y$ between two sup-lattices $X$ and $Y$, there exists its right adjoint $f_{*}: Y \rightarrow X$, which preserves arbitrary meets. The mapping $f_{*}$ can be defined explicitly as $f_{*}(y)=\bigvee\{x \in X \mid f(x) \leq y\}$.

The Cartesian product $\prod_{i \in I} X_{i}$ of posets $X_{i}, i \in I$, can be equipped with the standard product partial order $\left(x_{i}\right)_{i \in I} \leq\left(y_{i}\right)_{i \in I}$ iff $x_{i} \leq y_{i}$ for each $i \in I$. 


\subsection{Quantales}

A quantale is a sup-lattice $Q$ endowed with an associative binary operation (multiplication) · that distributes over arbitrary joins, i.e.,

$$
\begin{aligned}
& a \cdot \bigvee B=\bigvee_{b \in B}(a \cdot b) \\
& \bigvee B \cdot a=\bigvee_{b \in B}(b \cdot a)
\end{aligned}
$$

for any $a \in Q, B \subseteq Q$. A quantale $Q$ is unital if there is a unit element for multiplication, denoted by 1 . Distributivity of multiplication over arbitrary joins, including that of an empty set, implies that the least element $\perp$ also acts as a multiplicative zero. A quantale is commutative if the binary operation is commutative. If all elements of a quantale are idempotent, satisfying $x \cdot x=x$, we call the quantale idempotent.

For any element $q$ of a quantale $Q$, the unary operation $q \cdot-: Q \rightarrow Q$ is joinpreserving, therefore, having a (meet-preserving) right adjoint $q \rightarrow-: Q \rightarrow Q$, characterized by $q \cdot r \leq s \Longleftrightarrow r \leq q \rightarrow s$. Written explicitly, $q \rightarrow s=\bigvee\{r \in Q \mid$ $q r \leq s\}$.

Similarly, there is a right adjoint $q \leftarrow-: Q \rightarrow Q$ for $-\cdot q$, characterized by $r \cdot q \leq s \Longleftrightarrow r \leq q \leftarrow s$, and satisfying $q \leftarrow s=\bigvee\{r \in Q \mid r q \leq s\}$. If $Q$ is commutative, the operations $\rightarrow$ and $\leftarrow$ clearly coincide, and we will keep denoting them by $\rightarrow$.

Example 2.1. (1) The real unit interval $[0,1]$ with the standard partial order and multiplication of reals is a commutative quantale.

(2) The set of all open subsets of a topological space with unions as joins and binary meets as multiplication is a commutative idempotent quantale, called a frame or locale (frames are exactly commutative idempotent quantales).

(3) For any sup-lattice $S$, the set of its sup-lattice endomorphisms with mapping composition as multiplication and pointwise computed joins is a (generally noncommutative) quantale.

From the number of identities valid for commutative quantales, we will employ the following:

(1) $q \rightarrow \bigwedge r_{i}=\bigwedge\left(q \rightarrow r_{i}\right)$,

(2) $\bigvee q_{i} \rightarrow r=\bigwedge\left(q_{i} \rightarrow r\right)$,

(3) $\perp \rightarrow r=\top$,

(4) $1 \rightarrow r=r$.

\subsection{Categories}

For the required elements of category theory, the book [2] can be used for reference.

\section{Q-SUP-LATTICES}

Definition 3.1. By a base quantale we mean a unital commutative quantale $Q$. 
The base quantale is the structure in which $Q$-orders and $Q$-subsets are to be evaluated. For developing the theory in the rest of this paper, let $Q$ be an arbitrary base quantale that remains fixed from now on. Note that we do not require the multiplicative unit 1 of the base quantale to be its greatest element $T$, so there might be more values of 'being true' in $Q$.

\section{1. $Q$-ordered sets}

Definition 3.2. Let $X$ be a set. A mapping $e: X \times X \rightarrow Q$ is called a $Q$-order if, for any $x, y, z \in X$, the following are satisfied:

(1) $e(x, x) \geq 1$ (reflexivity),

(2) $e(x, y) \cdot e(y, z) \leq e(x, z)$ (transitivity),

(3) if $e(x, y) \geq 1$ and $e(y, x) \geq 1$, then $x=y$ (antisymmetry).

The pair $(X, e)$ is then called a $Q$-ordered set.

For a $Q$-order $e$ on $X$, the relation $\leq_{e}$ defined as $x \leq_{e} y \Longleftrightarrow e(x, y) \geq 1$ is a partial order in the usual sense. This means that any $Q$-ordered set can be viewed as an ordinary poset satisfying additional properties.

Vice versa, for a partial order $\leq$ on a set $X$ and any quantale $Q$, we can define a $Q$-order $e_{\leq}$by

$$
e_{\leq}(x, y)= \begin{cases}1, & \text { if } x \leq y \\ 0, & \text { otherwise }\end{cases}
$$

The definition of a $Q$-order implies that, for any $x, y \in X, e(x, y)=1 \cdot e(x, y) \leq$ $e(x, x) \cdot e(x, y) \leq e(x, y)$, i.e., the elements $e(x, x)$ act as local units with respect to other elements of the form $e(x, y)$, notably any $e(x, x)$ is idempotent.

Also note that any subset $Y$ of a $Q$-ordered set $\left(X, e_{X}\right)$ is itself a $Q$-ordered set with the restricted $Q$-order $e_{Y}=\left.e_{X}\right|_{Y}$.

Remark 3.3 (Q-duality principle). Let $\left(X, e_{X}\right)$ be a $Q$-ordered set. It can be easily verified that the mapping $e_{X}^{\mathrm{op}}(x, y)=e_{X}(y, x)$ is again a $Q$-order (the dual $Q$-order to $e_{X}$ ) on $X$. We denote the dual $Q$-ordered set to $X$ by $X^{\mathrm{op}}$.

Therefore, if a statement involving $Q$-orders is valid in all $Q$-ordered sets, then its dual statement, obtained by replacing the $Q$-orders with their duals, is valid in all $Q$-ordered sets too.

Definition 3.4. A $Q$-subset of a set $X$ is an element of the set $Q^{X}$.

For $Q$-subsets $M, N$ of a set $X$, we define the subsethood degree of $M$ in $N$ as

$$
\operatorname{sub}_{X}(M, N)=\bigwedge_{x \in X}(M(x) \rightarrow N(x))=\bigwedge_{x \in X}(\bigvee\{q \in Q \mid M(x) \cdot q \leq N(x)\}) .
$$

Then, $\left(Q^{X}, s u b_{X}\right)$ forms a $Q$-ordered set [1, Theorem 3.12]. In particular, $Q$ itself is a $Q$-ordered set with $e(x, y)=x \rightarrow y$.

Note that the definition of $Q$-subsets corresponds to viewing 'ordinary' subsets of $X$ as mappings from $X$ to the quantale $\mathbf{2}=\{0,1\}$.

Definition 3.5. Let $\left(X, e_{X}\right),\left(Y, e_{Y}\right)$ be $Q$-ordered sets.

- A mapping $f:\left(X, e_{X}\right) \rightarrow\left(Y, e_{Y}\right)$ is called $Q$-monotone if $e_{X}(x, y) \leq$ $e_{Y}(f(x), f(y))$. 
- Let $f:\left(X, e_{X}\right) \rightarrow\left(Y, e_{Y}\right), g:\left(Y, e_{Y}\right) \rightarrow\left(X, e_{X}\right)$ be $Q$-monotone mappings. We say that $(f, g)$ is a $Q$-adjunction, or a ( $Q$-monotone) Galois connection [10], if $e_{Y}(f(x), y)=e_{X}(x, g(y))$. Then, $f$ is called a left and $g$ a right $Q$-adjoint.

It is easy to show that the right and left $Q$-adjoint to a $Q$-monotone mapping $f$ are unique if they exist. Then, $f^{*}$ denotes the right $Q$-adjoint, and $f_{*}$ denotes the left $Q$-adjoint to $f$. From the definition, it also easily follows that when $f:\left(X, e_{X}\right) \rightarrow\left(Y, e_{Y}\right)$ and $g:\left(Y, e_{Y}\right) \rightarrow\left(Z, e_{Z}\right)$ are left-adjoint $Q$-monotone mappings, then $(g \circ f)^{*}=f^{*} \circ g^{*}$.

Remark 3.6. As with the definition of $Q$-ordered sets, it should be emphasized that:

- any $Q$-monotone mapping $\left(X, e_{X}\right) \rightarrow\left(Y, e_{Y}\right)$ is an ordinary monotone mapping $\left(X, \leq_{e_{X}}\right) \rightarrow\left(Y, \leq_{e_{Y}}\right)$,

- any $Q$-adjunction $(f, g)$ between $\left(X, e_{X}\right)$ and $\left(Y, e_{Y}\right)$ is a poset adjunction between $\left(X, \leq_{e_{X}}\right)$ and $\left(Y, \leq_{e_{Y}}\right)$.

Let $\left(X_{i}, e_{X_{i}}\right)$ for $i \in I$ be $Q$-ordered sets. On the set direct product $\prod_{i \in I} X_{i}$ we define a valuation

$$
e_{\underline{X}}\left(\left(x_{i}\right)_{i \in I},\left(y_{i}\right)_{i \in I}\right)=\bigwedge_{i \in I} e_{X_{i}}\left(x_{i}, y_{i}\right) .
$$

Indeed, $e_{\underline{X}}$ is a $Q$-order:

(1) $e_{\underline{X}}\left(\left(x_{i}\right)_{i \in I},\left(x_{i}\right)_{i \in I}\right)=\bigwedge_{i \in I} e_{X_{i}}\left(x_{i}, x_{i}\right) \geq 1$ as each $e_{X_{i}}\left(x_{i}, x_{i}\right) \geq 1$,

(2) $e_{\underline{X}}\left(\left(x_{i}\right)_{i \in I},\left(y_{i}\right)_{i \in I}\right) \cdot e_{\underline{X}}\left(\left(y_{i}\right)_{i \in I},\left(z_{i}\right)_{i \in I}\right)=\bigwedge_{i \in I} e_{X_{i}}\left(x_{i}, y_{i}\right) \cdot \bigwedge_{i \in I} e_{X_{i}}\left(y_{i}, z_{i}\right)$ $\leq e_{X_{i}}\left(x_{i}, y_{i}\right) \cdot e_{X_{i}}\left(y_{i}, z_{i}\right) \leq e_{X_{i}}\left(x_{i}, z_{i}\right)$ for any $i \in I$, therefore

$e_{\underline{X}}\left(\left(x_{i}\right)_{i \in I},\left(y_{i}\right)_{i \in I}\right) \cdot e_{\underline{X}}\left(\left(y_{i}\right)_{i \in I},\left(z_{i}\right)_{i \in I}\right)$

$\leq \bigwedge_{i \in I} e_{X_{i}}\left(x_{i}, z_{i}\right)=e_{\underline{X}}\left(\left(x_{i}\right)_{i \in I},\left(z_{i}\right)_{i \in I}\right)$

(3) $\left(e_{\underline{X}}\left(\left(x_{i}\right)_{i \in I},\left(y_{i}\right)_{i \in I}\right) \geq 1\right.$ and $\left.e_{\underline{X}}\left(\left(y_{i}\right)_{i \in I},\left(x_{i}\right)_{i \in I}\right) \geq 1\right)$

$\Longleftrightarrow\left(\bigwedge_{i \in I} e_{X_{i}}\left(x_{i}, y_{i}\right) \geq 1\right.$ and $\left.\bigwedge_{i \in I} e_{X_{i}}\left(y_{i}, x_{i}\right) \geq 1\right)$

$\Longrightarrow\left(e_{X_{i}}\left(x_{i}, y_{i}\right) \geq 1\right.$ and $\left.e_{X_{i}}\left(y_{i}, x_{i}\right) \geq 1\right)$ for any $i \in I$, therefore $x_{i}=y_{i}$ for any $i \in I$, hence $\left(x_{i}\right)_{i \in I}=\left(y_{i}\right)_{i \in I}$.

\section{2. $Q$-sup-lattices}

Definition 3.7. Let $M$ be a $Q$-subset of a $Q$-ordered set $(X, e)$. An element $s$ of $X$ is called a $Q$-join of $M$, denoted $\bigsqcup M$ if:

(1) $M(x) \leq e(x, s)$ for all $x \in X$, and

(2) for all $y \in X, \bigwedge_{x \in X}(M(x) \rightarrow e(x, y)) \leq e(s, y)$.

By analogy, an element $m$ of $X$ is called a $Q$-meet of $M$, denoted $\Pi M$, if:

(1) $M(x) \leq e(m, x)$ for all $x \in X$, and

(2) for all $y \in X, \bigwedge_{x \in X}(M(x) \rightarrow e(y, x)) \leq e(y, m)$.

Note that also in the $Q$-fuzzy setting, the concepts of joins and meets are dual a $Q$-join of $M$ with respect to the $Q$-order $e$ is a $Q$-meet of $M$ with respect to the dual ordering $e^{\text {op }}$. 
The following proposition provides an important characterization of $Q$-joins and $Q$-meets:

Proposition 3.8. Let $(X, e)$ be a $Q$-ordered set, $s, m \in X$ and $M \in Q^{X}$.

(1) $s=\bigsqcup M$ iff for all $y \in X, e(s, y)=\bigwedge_{x \in X}(M(x) \rightarrow e(x, y))$,

(2) $m=\prod M$ iff for all $y \in X, e(y, m)=\bigwedge_{x \in X}(M(x) \rightarrow e(y, x))$.

Proof. The proof is a slightly modified version of the one provided in [12], Theorem 2.2.

(1) (Necessity.) If $s$ is a $Q$-join of $M$, then for every $x \in X, M(x) \leq e(x, s)$. Therefore, for any $y \in Y$, we have $M(x) \cdot e(s, y) \leq e(x, s) \cdot e(s, y) \leq e(x, y)$, equivalently $e(s, y) \leq M(x) \rightarrow e(x, y)$, hence $e(s, y) \leq \bigwedge_{x \in X}(M(x) \rightarrow$ $e(x, y))$. Since the converse inequality is assumed by definition, we get an equality.

(2) (Sufficiency.) Suppose that for all $y \in Y, e(s, y)=\bigwedge_{x \in X}(M(x) \rightarrow e(x, y))$, and put $y=s$. Then $1 \leq e(s, s)=\bigwedge_{x \in X}(M(x) \rightarrow e(x, s))$, so for any $x \in X, 1 \leq M(x) \rightarrow e(x, s)$, equivalently $M(x)=M(x) \cdot 1 \leq e(x, s)$.

The inequality $\bigwedge_{x \in X}(M(x) \rightarrow e(x, y)) \leq e(s, y)$ follows from the assumption and $s$ is therefore a $Q$-join of $M$.

The antisymmetry property of the $Q$-order implies that, if a $Q$-join ( $Q$-meet) of a $Q$-subset exists, it is unique.

Example 3.9. Consider a $Q$-ordered set $(X, e)$ and its ' $Q$-empty subset' $x \mapsto \perp$. Then its join $s$ satisfies $e(s, y)=\bigwedge_{x \in X}(\perp \rightarrow e(x, y))=\bigwedge_{x \in X} \top=\top$ for any $y \in X$, and we denote it by $\perp_{X}$ if it exists. Similarly, for the meet $m$, we have $e(y, m)=\top$ for any $y \in X$, and we denote it by $\top_{X}$.

Indeed, $Q$-ordered sets having all $Q$-joins and all $Q$-meets behave very much like the usual complete lattices:

Definition 3.10. A $Q$-ordered set $\left(X, e_{X}\right)$ is called:

(1) $Q$-join-complete if $\bigsqcup M$ exists for any $Q$-subset $M$ of $X$,

(2) $Q$-meet-complete if $\prod M$ exists for any $Q$-subset $M$ of $X$,

(3) $Q$-complete if it is both $Q$-join-complete and $Q$-meet-complete.

Example 3.11. Using the definition of $Q$-joins and $Q$-meets, one can easily derive that a single-element $Q$-ordered set $(\{*\}, e)$ is $Q$-complete iff $e(*, *)=\top$. By Definition $3.7(1),. e(*, *) \geq M(*)$ for any $M \in Q^{\{*\}}$. Then $e(*, *)=\top$ clearly satisfies condition (2.), as $M(*) \rightarrow \top \leq \top$ always holds.

Proposition 3.12 ([10], Theorem 2.10, Corollary 2.11.). For a $Q$-ordered set $(X, e)$, the following are equivalent:

(1) $(X, e)$ is $Q$-join-complete.

(2) $(X, e)$ is $Q$-meet-complete.

(3) $(X, e)$ is $Q$-complete.

Proof. We omit the proof as it proceeds exactly in the same way as the one in [10], only considering the base quantale $Q$ rather than a complete residuated lattice $L$ (in a residuated lattice $1=\top$ ). 
Proposition 3.13. Let $(X, e)$ be a $Q$-complete $Q$-ordered set. Then $\left(X, \leq_{e}\right)$ is a complete poset, and, for any $S \subseteq X$, we have $\bigvee S=\bigsqcup \varphi_{S}$ where

$$
\varphi_{S}(x)= \begin{cases}1 & \text { if } x \in S, \\ \perp & \text { otherwise. }\end{cases}
$$

Proof. Denote $s=\bigsqcup \varphi_{S}$. Then, $1 \leq e(x, s)$ if $x \in S$, and for any $y \in X$,

$$
\begin{aligned}
e(s, y) & =\bigwedge_{x \in X}\left(\varphi_{S}(x) \rightarrow e(x, y)\right) \\
& =\bigwedge_{x \in S}\left(\varphi_{S}(x) \rightarrow e(x, y)\right) \\
& =\bigwedge_{x \in S}(1 \rightarrow e(x, y)) \\
& =\bigwedge_{x \in S} e(x, y) .
\end{aligned}
$$

For all $x \in S$, it is true that, if $y \geq_{e} x$, then $e(x, y) \geq 1$. It follows that $1 \leq \bigwedge_{x \in S} e(x, y)=e(s, y)$, hence, $y \geq_{e} s$.

Corollary 3.14. If $X$ is $Q$-complete, then $e(\bigvee S, y)=\bigwedge_{x \in S} e(x, y)$. By a dual argument, $e(x, \bigwedge S)=\bigwedge_{x \in S} e(x, y)$.

Proposition 3.15. If $X$ is $Q$-complete, then its $Q$-order $e(-,-)$ can be expressed by means of unary functions $e(x,-)$ resp. $e(-, x)$, i.e., for all $y, z \in X$, we have

$$
\begin{aligned}
& e(y, z)=\bigwedge_{x \in X}(e(x, y) \rightarrow e(x, z)), \\
& e(y, z)=\bigwedge_{x \in X}(e(z, x) \rightarrow e(y, x)) .
\end{aligned}
$$

Proof. We prove the first equality, the other follows from duality. Let $y, z \in$ $X$ be arbitrary, put $\varphi_{y} \in Q^{X}$ as $\varphi_{y}(x)=e(x, y)$, and denote $\bigsqcup \varphi_{y}(x)=x_{0}$. Then $e\left(x_{0}, z\right)=\bigwedge_{x \in L}\left(\varphi_{y}(x) \rightarrow e(x, z)\right)$. In particular, $\varphi_{y}\left(x_{0}\right)=e\left(x_{0}, y\right)=$ $\bigwedge_{x \in L}\left(\varphi_{y}(x) \rightarrow e(x, y)\right) \geq 1$.

We also have $\varphi_{y}(x) \leq e\left(x, x_{0}\right)$ for any $x \in X$, therefore, $1 \leq e(y, y)=\varphi_{y}(y) \leq$ $e\left(y, x_{0}\right)$, which implies $y=x_{0}$.

Since $Q$-joins and the mappings that preserve them will be of our primary interest, we will denote such $Q$-complete set by $\left(X, e_{X}\right)$ by $\left(X, \bigsqcup_{X}\right)$.

Definition 3.16. Let $X$ and $Y$ be sets, and $f: X \rightarrow Y$ a mapping. We define $Z$ adeh's forward power set operator [7] that maps $Q$-subsets of $X$ to $Q$-subsets of $Y$ as:

$$
f_{Q}(M)(y)=\bigvee_{x \in f^{-1}(y)} M(x) .
$$

There is also Zadeh's backward power set operator $f_{Q}^{\leftarrow}: Q^{Y} \rightarrow Q^{X}$ defined for $f$ as $f_{Q}^{\leftarrow}(M)=M \circ f$ 
Definition 3.17. Let $\left(X, e_{X}\right)$ and $\left(Y, e_{Y}\right)$ be $Q$-ordered sets. We say that a mapping $f: X \rightarrow Y$ is $Q$-join-preserving if, for any $Q$-subset $M$ of $X$ such that $\sqcup M$ exists, $\bigsqcup_{Y} f_{Q}(M)$ exists and

$$
f\left(\bigsqcup_{X} M\right)=\bigsqcup_{Y} f_{Q}(M)
$$

By analogy for $Q$-meet-complete sets, the mapping $f$ is $Q$-meet-preserving if, for any $Q$-subset $M$ of $X$ such that $\prod M$ exists, $\Pi_{Y} f_{Q}(M)$ exists and

$$
f\left(\prod_{X} M\right)=\prod_{Y} f_{Q}(M)
$$

The following proposition provides a tool useful for verifying that a mapping $f$ between $Q$-complete sets is $Q$-join-preserving. Rather than calculating with joins, one can check that $f$ is $Q$-monotone and find its right adjoint.

Proposition 3.18 ([10], Theorem 3.5). Let $\left(X, e_{X}\right),\left(Y, e_{Y}\right)$ be $Q$-ordered sets, and $f: X \rightarrow Y, g: Y \rightarrow X$ be two mappings. Then the following hold:

(1) If $\left(X, e_{X}\right)$ is $Q$-complete, then $f$ is $Q$-monotone and has a right adjoint iff $f(\bigsqcup M)=\bigsqcup f_{Q}(M)$ for all $M \in Q^{X}$.

(2) If $\left(Y, e_{Y}\right)$ is $Q$-complete, then $g$ is $Q$-monotone and has a left adjoint iff $g\left(\prod M\right)=\prod g_{Q}(M)$ for all $M \in Q^{Y}$.

Any $Q$-join-preserving mapping $f: X \rightarrow Y$ between $Q$-complete sets $\left(X, e_{X}\right)$ and $\left(Y, e_{Y}\right)$ thus has a $Q$-meet-preserving right adjoint $f^{*}: Y \rightarrow X$. This adjoint mapping can be also regarded (and we will often do so) as a $Q$-join-preserving mapping between the dual $Q$-ordered sets $Y^{\mathrm{op}}$ and $X^{\mathrm{op}}$. An analogous claim can be made for $Q$-meet-preserving mappings and their left adjoints.

In what follows, the $Q$-joins will mostly be discussed. Because of the apparent similarities between $Q$-join-complete sets and sup-lattices (we just recall that any $Q$-join complete set is actually a sup-lattice), we make a change in our terminology:

Definition 3.19. A $Q$-sup-lattice is a $Q$-join-complete set. Homomorphisms of $Q$-sup-lattices are $Q$-join-preserving mappings. By $Q$-Sup we will denote the category whose objects are $Q$-sup-lattices, and morphisms are $Q$-sup-lattice homomorphisms.

Definition 3.20. Let $(X, \sqcup)$ be a $Q$-sup-lattice, $Y \subseteq X$, and $M \in Q^{Y}$. We define an extension $M^{\prime} \in Q^{X}$ of $M$ as

$$
M^{\prime}(x)= \begin{cases}M(x) & \text { if } x \in Y, \\ \perp & \text { if } x \notin Y .\end{cases}
$$

We say that $Y$ is a sub-Q-sup-lattice of $X$ if it is closed under $Q$-joins in the following sense: for any $M \in Q^{Y}$, the element $\bigsqcup M^{\prime} \in X$ also belongs to $Y$.

The above definition has an equivalent statement: A set $Y \subseteq X$ is a sub-Q-suplattice of $X$ if, for any $M \in Q^{X}$ such that $M(x)=\perp$ for all $x \in X \backslash Y$, we also have $\bigsqcup M \in Y$.

Since the sub- $Q$-sup-lattice $Y$ can itself be regarded as a $Q$-sup-lattice, the inclusion mapping $Y \hookrightarrow Y$ is clearly a homomorphism. 


\subsection{Limits and colimits}

In Sup, both the product and the coproduct of a family of sup-lattices $X_{i}$ for $i \in I$ are carried by the Cartesian product $\prod_{i \in I} X_{i}$ which is a sup-lattice with joins calculated coordinatewise. We will show that, although $Q$-joins are not given simply at the individual coordinates, the product and coproduct (equalizer and coequalizer) constructions in the category $Q$-Sup are analogous to those in Sup. Having products available enables us to talk about the $Q$-sup-lattice of homomorphisms between two $Q$-sup-lattices.

Let $I$ be a set, $\left(X_{i}, \bigsqcup_{X_{i}}\right)$ be $Q$-sup-lattices for all $i \in I$, and $M$ be a $Q$-subset of the set $\prod_{i \in I} X_{i}$. For each $j \in I$ and $y \in X_{j}$, we then define $\widehat{M}_{j} \in Q^{X_{j}}$ as

$$
\widehat{M}_{j}(y)=\bigvee\left\{M\left(\left(x_{i}\right)_{i \in I}\right) \mid\left(x_{i}\right)_{i \in I} \in \prod_{i \in I} X_{i}, x_{j}=y\right\} .
$$

Proposition 3.21. Let $\left(X_{i}, \bigsqcup_{X_{i}}\right)$ be $Q$-sup-lattices for all $i \in I$. Then $\prod_{i \in I} X_{i}$ is a $Q$-sup-lattice too, with $\bigsqcup_{\underline{X}} M=\left(\bigsqcup_{X_{i}} \widehat{M}_{i}\right)_{i \in I}$.

Proof. Using Proposition 3.8, we show that the element $\left(\bigsqcup \widehat{M}_{i}\right)_{i \in I}$ is the $Q$-join of $M \in Q \prod X_{i}$. Let $\left(y_{i}\right)_{i \in I} \in \prod_{i \in I} X_{i}$, then

$$
\begin{aligned}
e_{\underline{X}}\left(\left(\bigsqcup \widehat{M}_{i}\right)_{i \in I},\left(y_{i}\right)_{i \in I}\right) & =\bigwedge_{j \in I} e_{X_{j}}\left(\bigsqcup \widehat{M}_{j}, y_{j}\right) \\
& =\bigwedge_{j \in I} \bigwedge_{x_{j} \in X_{j}}\left(\widehat{M}_{j}\left(x_{j}\right) \rightarrow e_{X_{j}}\left(x_{j}, y_{j}\right)\right) \\
& \left.=\bigwedge_{j \in I} \bigwedge_{x_{j} \in X_{j}}\left(\bigwedge_{\left(x_{i}\right) \in \prod_{i \neq j} X_{i}} M\left(\left(x_{i}\right)_{i \in I}\right)\right) \rightarrow e_{X_{j}}\left(x_{j}, y_{j}\right)\right) \\
& =\bigwedge_{j \in I} \bigwedge_{x_{j} \in X_{j}} \bigwedge_{\left(x_{i}\right) \in \prod_{i \neq j} X_{i}}\left(M\left(\left(x_{i}\right)_{i \in I}\right) \rightarrow e_{X_{j}}\left(x_{j}, y_{j}\right)\right) \\
& =\bigwedge_{j \in I} \bigwedge_{\left(x_{i}\right) \in \prod_{i} X_{i}}\left(M\left(\left(x_{i}\right)_{i \in I}\right) \rightarrow e_{X_{j}}\left(x_{j}, y_{j}\right)\right) \\
& =\bigwedge_{\left(x_{i}\right) \in \prod X_{i}} \bigwedge_{j \in I}\left(M\left(\left(x_{i}\right)_{i \in I}\right) \rightarrow e_{X_{j}}\left(x_{j}, y_{j}\right)\right) \\
& =\bigwedge_{\left(x_{i}\right) \in \prod X_{i}}\left(M\left(\left(x_{i}\right)_{i \in I}\right) \rightarrow \bigwedge_{j \in I}\left(e_{X_{j}}\left(x_{j}, y_{j}\right)\right)\right) \\
& \left.=\bigwedge_{\left(x_{i}\right) \in \prod X_{i}} M\left(\left(x_{i}\right)_{i \in I}\right) \rightarrow e_{\underline{X}}\left(\left(x_{i}\right)_{i \in I},\left(y_{i}\right)_{i \in I}\right)\right) \\
& =e_{\underline{X}}\left(\bigsqcup M,\left(y_{i}\right)_{i \in I}\right)
\end{aligned}
$$


We just note that, by $Q$-duality principle, an analogous claim holds for $Q$-meets.

Proposition 3.22. Let $\left(X_{i}, \bigsqcup_{X_{i}}\right)$ be $Q$-sup-lattices for all $i \in I$. Then their product is $\left(\prod_{i \in I} X_{i}, \bigsqcup_{\underline{X}}\right)$.

Proof. We show that the Cartesian product of $Q$-sup-lattices of the previous proposition satisfies the universal property of the product. Let $f_{i}: Z \rightarrow X_{i}$ for $i \in$ $I$ be $Q$-sup-lattice homomorphisms, and $\pi_{j}: \prod_{i \in I} X_{i} \rightarrow X_{j}, \pi_{j}\left(\left(x_{i}\right)_{i \in I}\right)=x_{j}$ be the projections. The universal mapping $g: Z \rightarrow \prod_{i \in I} X_{i}$ given as $g(z)=\left(f_{i}(z)\right)_{i \in I}$ satisfies $\pi_{j} \circ g=f_{j}$, and this is a unique way to define it. We need to check the following:

(1) All the projections $\pi_{j}: \prod_{i \in I} X_{i} \rightarrow X_{j}$ are homomorphisms.

Let $M \in Q^{\prod X_{i}}, j \in I, y \in X_{j}$. We have

$$
\pi_{j} \vec{Q}(M)(y)=\bigvee_{x \in \pi_{j}^{-1}(y)} M(x)=\bigvee\left\{M(x) \mid x \in \prod_{i \in I} X_{i}, x_{j}=y\right\}=\widehat{M}_{j}(y)
$$

Therefore,

$$
\pi_{j}(\bigsqcup M)=\pi_{j}\left(\left(\bigsqcup_{X_{i}} \widehat{M}_{i}\right)_{i \in I}\right)=\bigsqcup_{X_{j}} \widehat{M}_{j}=\bigsqcup_{X_{j}} \pi_{j Q}(M) .
$$

(2) The universal mapping $g: Z \rightarrow \prod_{i \in I} X_{i}, g(z)=\left(f_{i}(z)\right)_{i \in I}$ is a homomorphism.

Let $M \in Q^{Z}$. We show that $g(\bigsqcup M)=\left(f_{i}(\bigsqcup M)\right)_{i \in I}$ is the $Q$-join of $g_{Q}(M)$ in $\prod_{i \in I} X_{i}$, that is, for each $j \in I, f_{j}(\bigsqcup M)=\bigsqcup g_{\vec{Q}(M)}$. Fix any $j \in I$, and let $y \in X_{j}$ be arbitrary. Then,

$$
\begin{aligned}
& e_{X_{j}}\left(f_{j}(\bigsqcup M), y\right)=e_{X_{j}}\left(\bigsqcup f_{j} \rightarrow(M), y\right) \\
& =\bigwedge_{x \in X_{j}}\left(f_{j} \vec{Q}(M)(x) \rightarrow e_{X_{j}}(x, y)\right) \\
& =\bigwedge_{x \in X_{j}}\left(\left(\bigvee_{z \in f_{j}^{-1}(x)} M(z)\right) \rightarrow e_{X_{j}}(x, y)\right) \\
& =\bigwedge_{x \in X_{j}}\left(\left(\bigvee_{\left(x_{i}\right) \in \pi_{j}^{-1}(x)}\left(\bigvee_{z \in g^{-1}\left(\left(x_{i}\right)\right)} M(z)\right)\right) \rightarrow e_{X_{j}}(x, y)\right) \\
& =\bigwedge_{x \in X_{j}}\left(\left(\bigvee_{\substack{\left(x_{i}\right) \in \prod_{j} X_{i} \\
x_{j}=X_{i}}}\left(\bigvee_{z \in g^{-1}\left(\left(x_{i}\right)\right)} M(z)\right)\right) \rightarrow e_{X_{j}}(x, y)\right) \\
& =\bigwedge_{x \in X_{j}}\left(\left(\bigvee_{\substack{\left(x_{i}\right) \in \prod_{j} X_{i} \\
x_{j}=X_{i}}} g_{Q}(M)\left(\left(x_{i}\right)_{i \in I}\right)\right) \rightarrow e_{X_{j}}(x, y)\right)
\end{aligned}
$$




$$
=\bigwedge_{x \in X_{j}}\left(g_{\vec{Q}(M)_{j}}(x) \rightarrow e_{X_{j}}(x, y)\right)
$$

Proposition 3.23. The category $Q$-Sup has equalizers: Let $f, g: X \rightarrow Y$ be two homomorphisms. Then their equalizer is $\left(\left(X_{f g},\left.\bigsqcup_{X}\right|_{X_{f g}}\right), \varepsilon\right)$ where $X_{f g}=$ $\{x \in X \mid f(x)=g(x)\}$ and $\varepsilon: X_{f g} \rightarrow X$ is the set inclusion.

Proof. First we show that $X_{f g}$ is a sub-Q-sup-lattice of $X$ : let $M \in Q^{X_{f g}}$ be arbitrary, consider its extension $M^{\prime} \in Q^{X}$, which has its $\bigsqcup M^{\prime}$ in $X$. Then $f\left(\bigsqcup M^{\prime}\right)=\bigsqcup f_{Q}\left(M^{\prime}\right)=\bigsqcup g_{Q}\left(M^{\prime}\right)=g\left(\bigsqcup M^{\prime}\right)$ (since for any $y \in Y, f_{Q}\left(M^{\prime}\right)(y)=$ $\left.\bigvee\left\{M^{\prime}(x) \mid x \in X, f(x)=y\right\}=\bigvee\left\{M^{\prime}(x) \mid x \in X, g(x)=y\right\}=g_{Q}\left(M^{\prime}\right)(y)\right)$, thus $\bigsqcup M^{\prime}$ also belongs to $X_{f g}$. As we have already shown, the inclusion map is a homomorphism.

Let $h: Z \rightarrow X$ be a homomorphism satisfying $f \circ h=g \circ h$. Then $h(z) \in$ $X_{f g}$ for all $z$, so put $k: Z \rightarrow X_{f g}$ as $k(z)=h(z)$. Take $N \in Q^{Z}$ arbitrary, and check that $k$ is a homomorphism: $k(\bigsqcup N)=h(\bigsqcup N)=\bigsqcup h_{Q}(N)$. The element $s=\bigsqcup h_{\vec{Q}}(N) \in X$ satisfies for any $u \in X: e(s, u)=\bigwedge_{x \in X}\left(h_{\vec{Q}}(M)(x) \rightarrow\right.$ $\left.e_{X}(x, u)\right)=\bigwedge_{x \in X_{f g}}\left(h_{Q}(M)(x) \rightarrow e_{X}(x, u)\right)=\bigwedge_{x \in X_{f g}}\left(k_{Q}(M)(x) \rightarrow e_{X}(x, u)\right)$, hence $s=\bigsqcup k_{Q}(N) \in X_{f g}$.

The homomorphism $k$ is clearly defined in the only way to satisfy $\varepsilon \circ k=h$.

Corollary 3.24 ([2], Theorem 2.8.1). The category Q-Sup is complete.

Example 3.25. The category $Q$-Sup has a terminal object, the one-element $Q$-sup-lattice $\left(\{*\}, e_{*}\right)$ with $e_{*}(*, *)=\top$.

Proof. We have already shown in Example 3.11 that the ordering valuation $e(*, *)=\top$ is necessary in a single-element $Q$-sup-lattice. The constant mapping $x \mapsto *$ is clearly unique and $Q$-join preserving for any $Q$-sup-lattice $X$.

To demonstrate the cocompleteness of $Q$-Sup, we actually need not construct the desired objects and homomorphisms. Instead, we make use of adjoints and dual $Q$-orders. This is the same approach as can be found in [5, Section I.2] for sup-lattices.

Proposition 3.26. The category Q-Sup has coproducts.

Proof. Let $f_{i}: X_{i} \rightarrow Z, i \in I$ be homomorphisms. They all have their right adjoints $f_{i}^{*}: Z^{\mathrm{op}} \rightarrow X_{i}^{\mathrm{op}}$. We can take the product $\prod_{i \in I} X_{i}^{\mathrm{op}}$ with projections $\pi_{i}: \prod_{i \in I} X_{i}^{\mathrm{op}} \rightarrow X_{i}^{\mathrm{op}}$ for $i \in I$, so there is a homomorphism $g: Z^{\mathrm{op}} \rightarrow \prod_{i \in I} X_{i}^{\mathrm{op}}$ satisfying $\pi_{i} \circ g=f_{i}^{*}$ for any $i \in I$. Using the adjunctions again, we get a homomorphism $g^{*}:\left(\prod_{i \in I} X_{i}^{\mathrm{op}}\right)^{\mathrm{op}} \rightarrow\left(Z^{\mathrm{op}}\right)^{\mathrm{op}}=Z$ that satisfies $f_{i}=\left(\pi_{i} \circ g\right)^{*}=g^{*} \circ \pi_{i}^{*}$ for all $i \in I$.

We can verify that $\left(\prod_{i \in I} X_{i}^{\mathrm{op}}\right)^{\mathrm{op}}=\prod_{i \in I} X_{i}$ since $e_{\left(\prod X_{i}^{\mathrm{op}}\right)^{\mathrm{op}}}\left(\left(x_{i}\right)_{i \in I},\left(y_{i}\right)_{i \in I}\right)=$ $e_{\prod X_{i}^{\mathrm{op}}}\left(\left(y_{i}\right)_{i \in I},\left(x_{i}\right)_{i \in I}\right)=\bigwedge_{i \in I} e_{X_{i}^{\mathrm{op}}}\left(y_{i}, x_{i}\right)=\bigwedge_{i \in I} e_{X_{i}}\left(x_{i}, y_{i}\right)$ $=e_{\prod X_{i}}\left(\left(x_{i}\right)_{i \in I},\left(y_{i}\right)_{i \in I}\right)$. 
The homomorphisms $\iota_{j}=\pi_{j}^{*}: X_{j} \rightarrow \prod_{i \in I} X_{i}$ then provide the injections to the coproduct. Note that

$$
\iota_{j}(x)_{i}= \begin{cases}x & \text { if } i=j, \\ \perp_{X_{i}} & \text { otherwise }\end{cases}
$$

Proposition 3.27. The category Q-Sup has coequalizers.

Proof. Let $f, g: X \rightarrow Y$ be two homomorphisms. They both have their right adjoints $f^{*}, g^{*}: Y^{\mathrm{op}} \rightarrow X^{\mathrm{op}}$, so there exists the equalizer $\left(Y^{\mathrm{op}}\right)_{f^{*}, g^{*}}$ with a homomorphism $\varepsilon:\left(Y^{\mathrm{op}}\right)_{f^{*}, g^{*}} \rightarrow Y^{\mathrm{op}}$ satisfying the universal property. The mapping $\varepsilon$ also has its right adjoint $\varepsilon^{*}:\left(Y^{\mathrm{op}}\right)^{\mathrm{op}}=Y \rightarrow\left(\left(Y^{\mathrm{op}}\right)_{f^{*}, g^{*}}\right)^{\mathrm{op}}$, which yields the coequalizer of $f$ and $g$.

Corollary $\mathbf{3 . 2 8}$ ([2], dual to Theorem 2.8.1). The category Q-Sup is cocomplete.

Example 3.29. The category $Q$-Sup has an initial object, the one-element $Q$-sup-lattice $(\{*\}, e)$ with $e(*, *)=\top$.

Proof. By the construction presented above, the initial object as the coproduct of an empty system is identical to the empty product, i.e., the terminal object.

Note that the above statements imply that colimits in general are realized by the same objects as their corresponding limits they are dual to.

\subsection{Hom-objects}

For $Q$-sup-lattices $\left(X, \bigsqcup_{X}\right)$ and $\left(Y, \bigsqcup_{Y}\right)$, consider the set $\operatorname{Hom}(X, Y)$ of all homomorphisms $f:\left(X, \bigsqcup_{X}\right) \rightarrow\left(Y, \bigsqcup_{Y}\right)$. Then $\operatorname{Hom}(X, Y)$ becomes a $Q$-ordered set with

$$
e_{\mathrm{Hom}(X, Y)}(f, g)=\bigwedge_{x \in X} e_{Y}(f(x), g(x))
$$

(the restriction of product $Q$-order on $Y^{X}$ ). We will show that it is also a $Q$ sup-lattice (a sub- $Q$-sup-lattice of $Y^{X}$ ) with product $Q$-joins. We shall only verify that, for $M \in Q^{\operatorname{Hom}(X, Y)}$, the $Q$-join of its extension to $Y^{X}, \bigsqcup_{Y^{X}} M^{\prime}$, is a homomorphism too, that is, for any $N \in Q^{X}$,

$$
\left(\bigsqcup_{Y^{X}} M^{\prime}\right)\left(\bigsqcup_{X} N\right)=\bigsqcup_{Y}\left(\bigsqcup_{Y^{X}} M^{\prime}\right)_{Q}^{\rightarrow}(N)
$$

(1) First we characterize the $Q$-join of $M^{\prime} \in Q^{Y^{X}}$, call it $s$. Let $u \in X$, then $s(u)=\left(\bigsqcup_{Y^{X}} M^{\prime}\right)_{u}=\bigsqcup_{Y} \widehat{M}_{u}^{\prime}$ where, for $v \in Y, \widehat{M}_{u}^{\prime}(v)=\bigvee\left\{M^{\prime}(f) \mid f \in\right.$ $\left.Y^{X}, f(u)=v\right\}=\bigvee\{M(f) \mid f \in \operatorname{Hom}(X, Y), f(u)=v\}$.

By Proposition 3.8, we have, for any $y \in Y$,

$$
\begin{aligned}
e_{Y}(s(u), y) & =\bigwedge_{v \in Y}\left(\widehat{M}_{u}^{\prime}(v) \rightarrow e_{Y}(v, y)\right) \\
& =\bigwedge_{v \in Y}\left(\left(\bigvee_{\substack{X \rightarrow Y \\
f: X \rightarrow Y \\
f(u)=v}} M^{\prime}(f)\right) \rightarrow e_{Y}(v, y)\right)
\end{aligned}
$$




$$
\begin{aligned}
& =\bigwedge_{v \in Y}\left(\bigwedge_{\substack{X: X \rightarrow Y \\
f(u)=v}}\left(M^{\prime}(f) \rightarrow e_{Y}(v, y)\right)\right) \\
& =\bigwedge_{f: X \rightarrow Y}\left(M^{\prime}(f) \rightarrow e_{Y}(f(u), y)\right) \\
& =\bigwedge_{f \in \operatorname{Hom}(X, Y)}\left(M(f) \rightarrow e_{Y}(f(u), y)\right)
\end{aligned}
$$

(2) We make use of Proposition 3.18 and demonstrate an equivalent property that $s=\bigsqcup_{X^{Y}} M$ is $Q$-monotone and has a right adjoint.

(a) Let $x_{1}, x_{2} \in X$. Then, by the derivation above,

$$
e_{Y}\left(s\left(x_{1}\right), s\left(x_{2}\right)\right)=\bigwedge_{f \in \operatorname{Hom}(X, Y)}\left(M(f) \rightarrow e_{Y}\left(f\left(x_{1}\right), s\left(x_{2}\right)\right)\right) .
$$

As any $f \in \operatorname{Hom}(X, Y)$ satisfies $M(f) \leq e_{\left(Y^{X}\right)}(f, s)$

$=\bigwedge_{x \in X} e_{Y}(f(x), s(x))$, we have $M(f) \leq e_{Y}\left(f\left(x_{2}\right), s\left(x_{2}\right)\right)$. Hence,

$$
\begin{aligned}
e_{X}\left(x_{1}, x_{2}\right) \cdot M(f) & \leq e_{X}\left(x_{1}, x_{2}\right) \cdot e_{Y}\left(f\left(x_{2}\right), s\left(x_{2}\right)\right) \\
& \leq e_{Y}\left(f\left(x_{1}\right), f\left(x_{2}\right)\right) \cdot e_{Y}\left(f\left(x_{2}\right), s\left(x_{2}\right)\right) \\
& \leq e_{Y}\left(f\left(x_{1}\right), s\left(x_{2}\right)\right),
\end{aligned}
$$

which is equivalent to $e_{X}\left(x_{1}, x_{2}\right) \leq M(f) \rightarrow e_{Y}\left(f\left(x_{1}\right), s\left(x_{2}\right)\right)$, and therefore $e_{X}\left(x_{1}, x_{2}\right) \leq e_{Y}\left(s\left(x_{1}\right), s\left(x_{2}\right)\right)$.

(b) Any $g \in \operatorname{Hom}\left(Y^{\mathrm{op}}, X^{\mathrm{op}}\right)$ has a unique left adjoint $f \in \operatorname{Hom}(X, Y)$, so put $M^{*}: \operatorname{Hom}\left(Y^{\mathrm{op}}, X^{\mathrm{op}}\right) \rightarrow Q$ as $M^{*}(g)=M(f)$. Then, for any $y \in Y$, we obtain

$$
\begin{aligned}
e_{Y}(s(x), y) & =\bigwedge_{f \in \operatorname{Hom}(X, Y)}\left(M(f) \rightarrow e_{Y}(f(x), y)\right) \\
& =\bigwedge_{g \in \operatorname{Hom}\left(Y^{\mathrm{op}}, X^{\mathrm{op}}\right)}\left(M^{*}(g) \rightarrow e_{X}(x, g(y))\right) \\
& =\bigwedge_{g: Y_{Y}^{\mathrm{op}} \rightarrow X^{\mathrm{op}}}\left(M^{*^{\prime}}(g) \rightarrow e_{X}(x, g(y))\right) \\
& =e_{X}\left(x,\left(\prod M^{*}\right)(y)\right),
\end{aligned}
$$

and $\bigsqcup M^{*}$ is thus the right adjoint to $s=\bigsqcup M$. By the same procedure as in step 2. (a), we can verify that $\Pi M^{*}$ is $Q$-monotone too.

Corollary 3.30. For $Q$-sup-lattices $X$ and $Y$, the set $\operatorname{Hom}(X, Y)$ is also a $Q$ sup-lattice.

Example 3.31. A $Q$-quantale is a $Q$-sup-lattice $A$ endowed with an associative binary operation - which is $Q$-join-preserving in both components, namely for any $a \in A$ and $M \in Q^{A}$,

$$
\begin{aligned}
& (\bigsqcup M) \cdot a=\bigsqcup(-\cdot a)_{Q}(A) \\
& a \cdot(\bigsqcup M)=\bigsqcup(a \cdot-) \vec{Q}(A)
\end{aligned}
$$


$(Q$-quantales are semigroup objects in $Q$-Sup as quantales are semigroup objects in Sup.) As a common example of $Q$-quantales, a commutative quantale $Q$ with $e(x, y)=x \rightarrow y$ is typically presented [13, Remark 4.4]. We are now able to provide another instance: Let $X$ be a $Q$-sup-lattice, then the set of all its endomorphisms $\operatorname{End}(X)=\operatorname{Hom}(X, X)$ is a $Q$-quantale with composition of mappings as the multiplication. We just need to verify that $\bigsqcup(g \circ-)_{Q}(M)=g \circ \bigsqcup M$ for any $M \in Q^{\operatorname{End}(X)}$. Let $k \in \operatorname{End}(X)$ be arbitrary, and calculate:

$$
\begin{aligned}
& e_{\operatorname{End}(X)}\left(\bigsqcup(g \circ-)_{Q}(M), k\right)=\bigwedge_{h \in \operatorname{End}(X)}\left((g \circ-) \vec{Q}(M)(h) \rightarrow e_{\operatorname{End}(X)}(h, k)\right) \\
& =\bigwedge_{h \in \operatorname{End}(X)} \bigwedge_{\substack{f \in \operatorname{End}(X) \\
g \circ f=h}}\left(M(f) \rightarrow e_{\operatorname{End}(X)}(h, k)\right) \\
& =\bigwedge_{h \in \operatorname{End}(X)} \bigwedge_{\substack{f \in \operatorname{End}(X) \\
g \circ f=h}} \bigwedge_{x \in X}\left(M(f) \rightarrow e_{X}(h(x), k(x))\right) \\
& =\bigwedge_{f \in \operatorname{End}(X)} \bigwedge_{x \in X}\left(M(f) \rightarrow e_{X}(g(f(x)), k(x))\right) \\
& =\bigwedge_{x \in X} \bigwedge_{f \in \operatorname{End}(X)}\left(M(f) \rightarrow e_{X}(g(f(x)), k(x))\right) \\
& =\bigwedge_{x \in X} \bigwedge_{f \in \operatorname{End}(X)}\left(M(f) \rightarrow e_{X}\left(f(x), g^{*}(k(x))\right)\right) \\
& =\bigwedge_{x \in X} e_{X}\left((\bigsqcup M)(x), g^{*}(k(x))\right) \\
& =\bigwedge_{x \in X} e_{X}(g((\bigsqcup M)(x)), k(x)) \\
& =\bigwedge_{x \in X} e_{X}((g \circ \bigsqcup M)(x), k(x)) \\
& =e_{\operatorname{End}(X)}(g \circ \bigsqcup M, k)
\end{aligned}
$$

The other equality, $\bigsqcup(-\circ g)_{Q}(M)=\bigsqcup M \circ g$, can be verified by the same procedure.

Acknowledgement. The author would like to thank his advisor Jan Paseka for introducing the topic and providing many valuable hints and comments.

\section{REFERENCES}

[1] R. Bělohlávek, Fuzzy Relational Systems, Foundations and Principles, IFSR International Series on Systems Science and Engineering, Springer, 2002.

[2] F. Borceux, Handbook of Categorical Algebra, Volume 1, Basic Category Theory, Encyclopedia of Mathematics and its Applications 50, Cambridge University Press, 2008.

[3] L. Fan, A new approach to quantitative domain theory,Electron. Notes Theor. Comput. Sci. 45 (2001), 77-87. 
[4] B. Flagg, P. Sünderhauf and K. Wagner, A logical approach to quantitative domain theory, Topology Atlas Preprint 23, preprint submitted to Elsevier Preprint (1996), 29 pp.

[5] A. Joyal, M. Tierney, An Extension of the Galois Theory of Grothendieck, Mem. Am. Math. Soc. 51, 1984.

[6] J. Paseka and D. Kruml, Algebraic and categorical aspects of quantales, in: M. Hazewinkel, Handbook of Algebra 5, North-Holland, Elsevier, 2008, 323-362.

[7] S. E. Rodabough, Powerset operator foundations for poslat fuzzy set theories and topologies, in: U. Höhle et al. (eds.), Mathematics of Fuzzy Sets. The Handbooks of Fuzzy Sets Series 3 , Springer, 1999, 91-116.

[8] K. I. Rosenthal, Quantales and Their Applications, Longman Scientific \& Technical, 1990.

[9] S. A. Solovyov, Quantale algebras as a generalization of lattice-valued frames, to appear in Hacet. J. Math. Stat.

[10] W. Yao and L.-X. Lu, Fuzzy Galois connections on fuzzy posets, Math. Log. Q. 55 (2009), 105-112.

[11] L. A. Zadeh, Fuzzy Sets, Inf. Control 8 (1965), 338-353.

[12] Q. Zhang, W. Xie and L. Fan, Fuzzy complete lattices, Fuzzy Sets Syst. 160 (2009), 22752291.

[13] B. Zhao, S. Wu and K. Wang, Quantale algebras as lattice-valued quantales, Soft Comput. (2016), first online 20 April 2016, 14 pp.

Radek Šlesinger, Department of Mathematics and Statistics, Faculty of Science, Masaryk University, Kotlářská 2, 61137 Brno, Czech Republic

e-mail: xslesing@math.muni.cz 
